\title{
Transmission Line Intelligent Inspection System Based on
}

\author{
GIS \\ Changchun ZOU Qingyue LUO Xiaofang WANG Meimei LUO Baiyang \\ LIU \\ (Department of Electrical Engineering, Shaoyang University, Shaoyang 422004, Hunan) \\ 651954331@qq.com
}

Keywords: intelligent transmission line inspection system; GIS; handheld intelligent terminal Abstract. Approach presents an intelligent inspection system for transmission line based on GIS(Geographic Information System). The construction principle, system structure and function of the intelligent inspection system are introduced in detail. The basic idea of the system is to embed GIS handheld intelligent terminal, via GPS (Global Positioning System), BDS (BeiDou Navigation Satellite System), GPRS wireless communication technology, the inspection data is imported into the cloud system in real-time, so managers can in the client computer, real-time view inspection status, issued inspection instructions, to achieve a visual inspection, information, intelligence.

\section{Introduction}

Transmission line as the middle part of power system, exposure to natural environment, in addition to the normal mechanical and electrical load to bear, still need to bear filth, lightning, torrential flood, bird damage, earthquake, high winds and other external infringement. These violations will have the potential to cause damage, deterioration, fatigue, corrosion of transmission line components, If not found in time to repair, it will threaten the safety and stability of power system. In order to maintain the normal operation of the transmission line, at present, direct labeling, bar code identification, information acquisition method, RF acquisition method, helicopter method, and robot method are widely used in China. The characteristics of the several polling mode are as following: (1) Although the low cost of the technology, the efficiency is also low , and management not convenient ; (2) some technologies built on proprietary platforms, the high cost is not conducive to the promotion ; (3) some techniques have too many human factors, and the effect of inspection and assessment is affected ${ }^{[1-2]}$.

This paper is based on the original line inspection, integrated GIS, GPS, GPRS and Beidou navigation four technologies proposed to build a GIS based, Beidou navigation, GPS double star positioning, GPRS, Beidou communication dual communication intelligent, visual transmission line intelligent patrol system ${ }^{[3]}$.The advantages are as follows: (1) Eliminating construction and maintenance. Do not need to be installed on transmission lines or towers information identifying the carrier, using automatic timing positioning of electronic maps and GPS; (2) reduce the absenteeism 
inspection, reducing inspection personnel work intensity, reduce system cost. Through fingerprint identification and positioning communication function of intelligent handheld terminals, real-time updates inspection schedule, inspection data entry in the handheld terminal, easy to standardize management; (3) increase the accuracy of the timeliness of the defect eliminated. To realize the full and efficient supervision from the defect discovery to the defect removal, reduce undetected and error detection times ${ }^{[4]}$.

\section{System Construction Principles}

The following principles should be followed in the design and development of transmission line intelligent inspection system:

1) Advance: integrated GIS, navigation, and network communication technology, real-time tracking inspection, inspection data in real-time update, to achieve paperless inspection, visualization, and can query the historical defects, to accept the instruction, timely defect elimination.

2) Reliability: The system is based on GPS and Beidou navigation positioning time, can improve the positioning accuracy and speed effectively, and can be in the absence of GPS, using Beidou autonomous positioning system, using GPRS and Beidou dual communication mode at the same time, areas the network resources in good to use GPRS communications, in the mountains and other communications blackout use Beidou communication, and can send emergency signals in case of emergency, high reliability.

3) Scalability: In order to meet the requirements of the future development of modular, the system building process, fully considering the future access to other functions, set aside enough interface, to avoid "technology island" phenomenon, and make full use of system efficiency.

4) Practicality: Equipment constitutes a low cost, mature technology, no need to install and maintain information identification device on the transmission line or the tower, based on user-friendly design of handheld intelligent terminal provide a graphical user interface, simple operation, easy to use.

\section{System structure}

\section{System components}

Transmission line intelligent inspection system is composed of three parts, intelligent handheld terminal, cloud server and the computer client. Fig. 1 is a topology of system architecture. With the mobile wireless communication network, the handheld intelligent terminal transmits the inspection data and satellite positioning trajectory data to the cloud server, cloud server to update the data in real time, managers can view real-time inspection information through computer client. The client and the server to communicate with the cloud server through TCP/IP. When the wireless communication does not cover the area or in case of emergency, the handheld intelligent terminal can communicate directly with the Beidou satellite, sending simple inspection information or distress signal. 

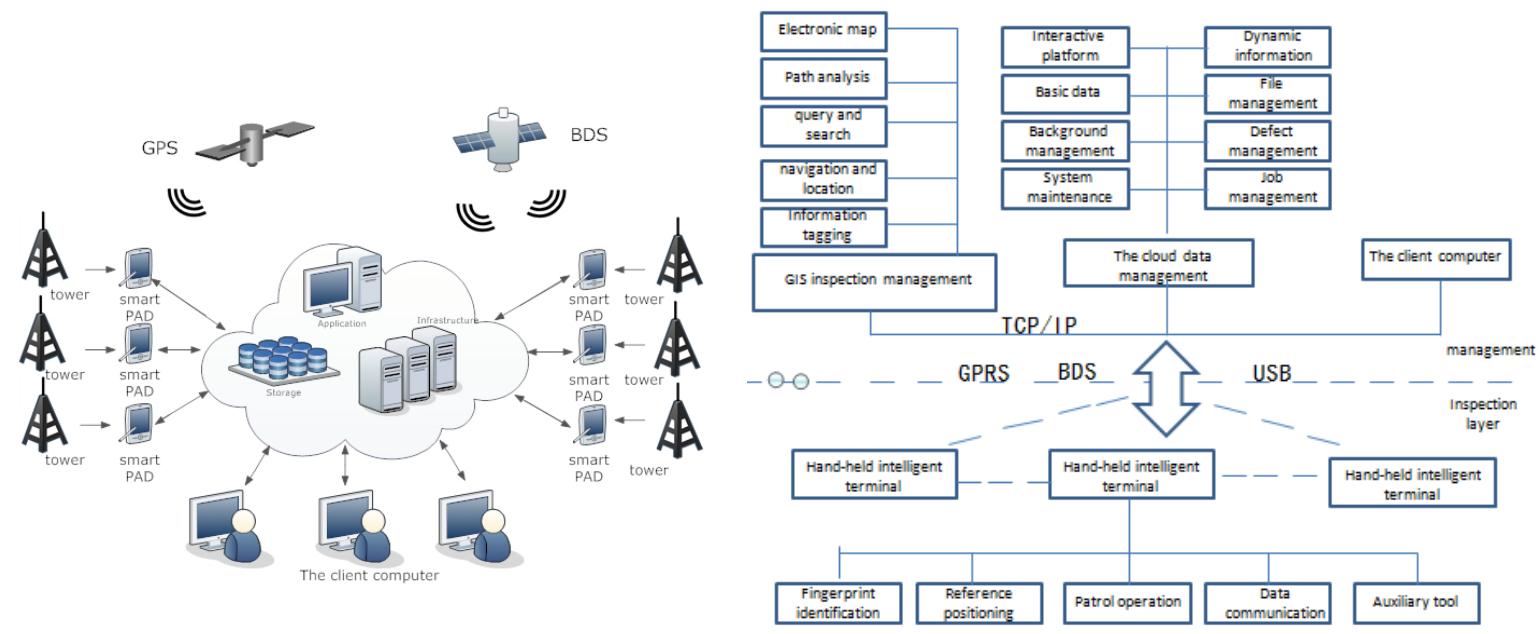

Fig.1 Transmission Line Intelligent Inspection System Topology

Fig. 2 Transmission Line Intelligent Inspection System Architecture

\section{System architecture}

When the system architecture framework, taking into account the cloud server and computer clients are in the back end, the implementation of system center management is defined as management level; intelligent handheld terminal bears the specific inspection work, defined as inspection layer, as shown in Fig. 2.

Eight subsystems the functions of PC client and cloud server the co-processing system, including: dynamic information, records management, defect management, operation management, interactive platform, basic data, backstage management, system maintenance; GIS inspection information management system can realize the visualization of the inspection work in the computer terminal and the handheld intelligent inspection terminal, its electronic map, path analysis, navigation and positioning, information tagging and other functions, greatly improve the efficiency of transmission line inspection of field operations, reduce the work intensity of the inspection staff, ensure inspection quality and scientific and efficient management of inspection work.

\section{System functions}

The system is composed of handheld intelligent terminal, cloud server and computer client. Where handheld intelligent terminal for collecting inspection data, belonging to the front; cloud server and computer client to carry out the data stored in the handheld intelligent terminal, storage and processing, and other processing, belonging to the backend.

\section{Handheld intelligent terminal}

1) Fingerprint identification. The three main factors that affect the transmission line working correctly or not, one of the factors that patrol officers. Mainly in two points: First, if inspection in place; the second, whether there an alternative inspection or not. Previous inspection methods, can not be eliminated from the root causes of the above two cases, by adding the fingerprint recognition function in the handheld intelligent terminal, the unique biological properties of the fingerprint are used ${ }^{[5]}$, with GIS electronic map and GPS, Beidou navigation, when the patrol officers arrived patrol point, the handheld intelligent terminal will be the voice and the vibrating alert patrol officers, fingerprint entry, carry out inspection work. This would solve the situation from the source of the inspection staff is not in place, and without the permission to find someone else to replace.

2) Reference location. In order to ensure the quality of the transmission line inspection, the inspection personnel are required to carry out inspection work along the transmission line. Use Beidou or GPS positioning and navigation functions in real-time on the electronic map GIS to form of transmission line inspection track, the system according to the "shortest distance" calculation method ${ }^{[6]}$ analyze the path of inspection personnel, when the inspection personnel departing from 
the inspection points or the inspection route, can remind the inspection personnel in vibration and voice. If wild lost in the wild, intelligent planning inspection paths or escape route.

Fig. 3 Transmission line inspection workflow

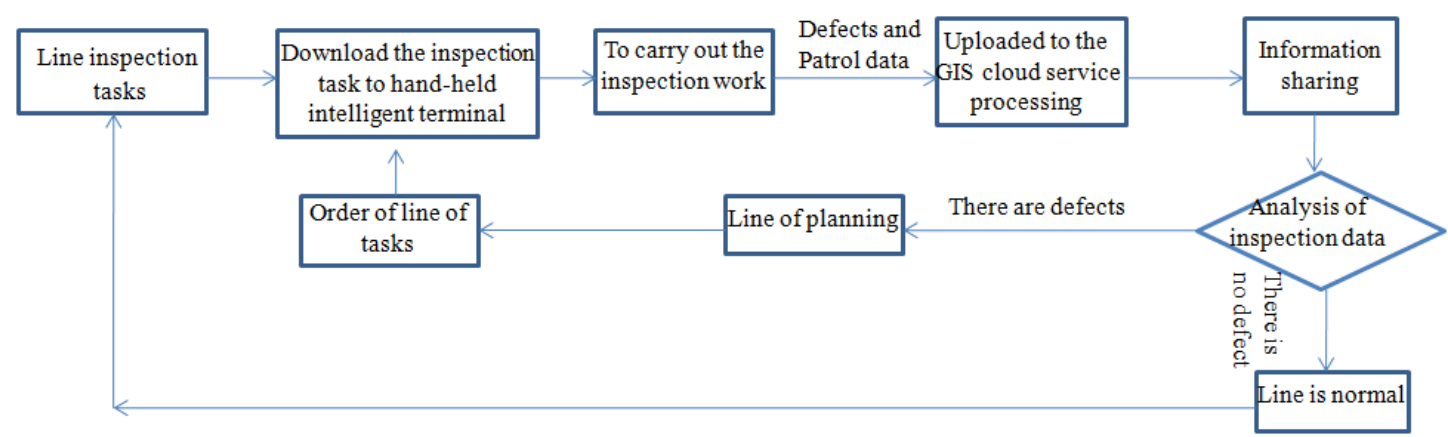

3) Patrol line operation. Inspection of the custom route or area according to the current inspection task: Download and open the map file to execute the task in the intelligent handheld terminal, used as a patrol base; according to the task route and equipment inspection scope of the given map, navigation inspection personnel to form the inspection tasks, record the inspection results data and photo evidence, workflow of the inspection are shown in Fig. 3; automatically collect the coordinates of the inspection personnel and upload to the cloud server, if the inspection path is not the task one, the task of the computer client management layer is received and the inspection path adjusted; the inspector may select the defect type grade of the intelligent terminal based on the specific defects of the line and equipment, as inspection data and the scene does not match, the inspector can manually write the inspection results, and through the wireless data communications uploaded to the cloud server save for later use ${ }^{[7]}$.

4 ) Data communication. Handheld intelligent terminal through the GPRS communication module to communicate with the cloud server, uploading and downloading inspection data; at the same time, the computer client can also communicate with the cloud server, to see and communicate relevant instructions. When the GPRS communication is interrupted, the system will automatically call the Beidou communications as a standby communication, through simple text, delivery instructions, while the inspection image and audio data stored in the intelligent terminal, and wait until GPRS signal is restored, uploaded to the cloud server, or through a USB data line upload data.

5 ) Auxiliary tool. Other programs on the system to assist, including special tips, such as patrol and patrol the tower to do different identification; battery power display; battery power display of the intelligent handheld terminal. Satellite guiding, that is, by entering the position data you can get the distance and direction between two points. Blotter, namely the non inspection defect information to provide notepad to be recorded, stored ${ }^{[4]}$.

\section{Cloud server}

Cloud server based on cloud computing, cloud computing is a large-scale distributed computing model; through the cloud can form an abstract, virtual, dynamically scalable resource pool, the resource pool can provide computing power to users on demand via Internet, storage capacity, the development platform and software services ${ }^{[8]}$.

The main function of the cloud service control center is based on the request of the user, the user's request tasks are divided into several sub tasks, and then dynamically assigning each sub task to the computing device integrated with the cloud computing platform by Internet. In addition, the 
cloud service control center is also responsible for storing the data through the Internet to the data storage device, and the need to re-read the data from the storage device. Using virtualization and segmentation techniques, the scheduling and allocation of the tasks for the calculation and data storage are completely invisible to the user. Cloud computing platform for each user to create a completely independent virtual system environment, so, in every user's view, they are the only cloud computing platform. Users can through a variety of different terminals, such as: handheld intelligent terminal, desktop computer, portable computer. Cloud service platform is very convenient to use ${ }^{[9]}$.

\section{PC Client}

Management client can achieve line inspection of senior management, system management, project management, patrol line management, defect management, data communications, report printing, auxiliary functions and extensions. Realization of visual intelligent and efficient line inspection management.

\section{System Features}

Features are as follows: (1) Join track automatic tracking analysis in GIS electronic map ; (2) data transmission in the original serial communication to the BDS and GPRS real-time dual communication mode; (3) on the basis of original positioning module and communication module, handheld terminal added fingerprint identification module; (4) into the cloud data storage and processing functions.

\section{Conclusion}

Applied GIS technology to the transmission line inspection and designed the intelligent inspection system of transmission line, which can achieve wide-area transmission line geospatial data management and maintenance. And the GPS and Beidou navigation technology real-time positioning and monitoring of the field patrol inspection personnel, at the same time to ensure that the inspection staff by the inspection task to develop the work path for inspection,intelligently record the actual inspection personnel patrol paths and the path of real time and task is compared, guidelines on inspection personnel according to the correct path, the right direction inspection, avoid duplication of inspection and undetected,To avoid the occurrence of accidents caused by the wrong examination. The system is in line with the business process of electric power inspection, and has a good application prospect.

\section{References}

[1]Lan Xue. Research and Design of Helicopter Transmission Line Inspection System Based on GIS [D]. Harbin: Harbin University of Science and Technology,.2008:1-61

[2]Wenjie Wang. Research on Transmission Line Intelligent Inspection System Based on GIS-GPS [D]. Wuhan: Wuhan University of Technology.2012:1-51

[3]Jiajia Zhu. Design and Implementation of Electric Power Line Inspection System Based on GIS [J]. Electrical Applications, 2015,34 (10).141-144

[4]Yi Hu. Kai Liu. Remote Sensing Inspection and Detection Technology for Transmission Line [M]. Beijing, China Power Press 2012,10

[5]Chikin Wan. Fingerprint Image Recognition Technology and Its Application Prospects [J]. 
Computer and Communications, 2003.21 ( 2 ) .14-16

[6]Chunxia Hu. Suzhen.Wang Chenglong Sun. GPS-based Transmission Line Inspection [J].

Computer Technology and Development, 2012.22 (6 ) .175-178

[7]Suzhen Wang. Zhenhua Xia. Shaokai Sun. Dehua Zhang. Liu Shukun. Research on High Voltage

Transmission Line Inspection System Based on GIS Technology [J]. Computer Technology and Development, 2012.22 (6) .175-178

[8]FOSTER I,ZHAO Y,RAICU I, et al.Cloud computing and grid computing 360-degree compared//Proceedings of Grid Computing Environments Workshop,November 12-16,2008,Austin,TX,USA:1-10.

[9]Junhua Zhao.Fushuan Wen. Yusheng Xue. Zhilin Zhen Cloud Computing: Constructing the Future of Computing Power System Core Platform [J]. Automation of Electric Power Systems, $2010.34(15) .1-8$ 